was not increased in children who did not receive penicillin in periods up to three hours taken to reach hospital. This suggests that a short delay without penicillin is not deleterious.

Why did the original paper by Cartwright et $\mathrm{al}^{1}$ (and a contemporaneous paper by Strang and Pugh ${ }^{10}$ ) suggest a positive benefit from penicillin when we and our Danish colleagues show such a clear association with poor outcomes? One contributory explanation for the difference lies in the inclusion or exclusion of children in whom the general practitioner did not make the diagnosis of meningococcal disease and who were therefore unlikely to receive penicillin before admission. If we had included the 166 children who were seen but not diagnosed by general practitioners and who reached hospital at a median of 12 hours later without having parenteral penicillin before admission, the estimated crude odds of death after penicillin would be reduced from 5.96 to 1.45 , at the upper limit of the $95 \%$ confidence interval of the estimate by Cartwright et al. ${ }^{1}$ The current discrepancies can be answered only by a randomised controlled trial.

Robert Booy contributed substantially to the design of the original study. Clare Phillips assisted with the data collection.

Contributors: See bmj.com.

Funding: The data were collected in conjunction with the research division of the Royal College of Paediatrics and Child Health. The project was funded by the Meningitis Research Foundation which received a grant from the National Lottery Charities Board (now the Big Lottery Fund), which enabled support for part of this study. The research team in Oxford is supported by the UK Medical Research Council.

Competing interests: None declared

Ethical approval: South Thames multi-research ethics committee and all local research ethics committees in England, Wales, and Northern Ireland.

1 Cartwright K, Reilly S, White D, Stuart J. Early treatment with parentera penicillin in meningococcal disease. BMJ 1992;305:143-7.

2 Sorensen HT, Nielsen GL, Schonheyder HC, Steffensen FH, Hansen I, Sabroe S, et al. Outcome of pre-hospital antibiotic treatment of meningococcal disease. J Clin Epidemiol 1998;51:717-21.

\section{What is already known on this topic}

Current guidance to UK general practitioners is to give penicillin to children with suspected meningococcal infection before they are admitted to hospital

\section{What this study adds}

Children who receive penicillin before admission have more severe disease on reaching hospital

Children given penicillin may have had more severe disease when they were first seen by a general practitioner

An adverse effect of penicillin in the first hour cannot be excluded, though this cannot be explained by our current understanding of biological mechanisms

3 Norgard B, Sorensen HT, Jensen ES, Faber T, Schonheyder HC, Nielsen GL. Pre-hospital parenteral antibiotic treatment of meningococcal disease and case fatality: a Danish population-based cohort study. J Infect 2002;45:144-51.

4 Ninis N, Philips C, Bailey L, Pollock JI, Nadel S, Britto J, et al. The role of healthcare delivery in the outcome of meningococcal disease in children:
case control study of fatal cases and survivors. BMJ 2005;330:1475-8.

5 Mellado M, Rodriguez-Contreras R, Mariscal A, Luna J, DelagadoRodriguez M, Galvez-Varges R. Effect of penicillin and chloramphenicol on the growth and endotoxin release by N meningitidis. Epidemiol Infect 1991;106:283-8

6 Hurley JC. Antibiotic-induced release of endotoxin: a reappraisal. Clin Infect Dis 1992;15:840-54.

7 Brandtzaeg P, Kierulf P, Gaustad P, Skulberg A, Bruun JM, Halvorsen S, et al. Plasma endotoxin as a predictor of multiple organ failure and death in systemic meningococcal disease. J Infect Dis 1989;159:195-204.

8 Andersen B, Solberg O. The endotoxin-liberating effect of antibiotics on meningococci in vitro. Acta Pathol Microbiol Scan 1980;88:231-6.

9 Brandtzaeg P, Kierulf P, Gaustad P, Dobloug J, MollnesTE, Sirnes K. SysBrandtzaeg P, Kierulf P, Gaustad P, Dobloug J, MollnesTE, Sirnes K. Sys-
temic meningococcal disease: a model infection to study acute endotoxtemic meningococcal disease: a model infection to
inemia in man. Prog Clin Biol Res 1988;272:263-71.

10 Strang J, Pugh E. Meningococcal infections: reducing the case-fatality rate by giving penicillin before admission to hospital. BMJ 1992;305:141-3. (Accepted 23 February 2006)

doi $10.1136 /$ bmj.38789.723611.55

\title{
Commentary: Statistics and death from meningococcal disease in children
}

\author{
Rafael Perera
}

Medical statisticians seldom directly make life and death decisions. Though I wouldn't like to have direct responsibility for making the decision to give a penicillin injection to a child with a purpuric rash in the community, I am conscious of the effect that my work may have on clinical decisions for such children. I felt a heavy responsibility when I conducted the statistical analysis of this paper. ${ }^{1}$

In most datasets that I analyse, the main issue is to quantify if the effect observed is due to chance. The question is rarely about the direction of the main effect; instead it is more about the size of the effect and the precision with which it has been estimated. But on this occasion one key statistical decision determined whether the best estimate of the effect of parenteral penicillin given before admission to hospital was a modest benefit or substantial harm (table), and the statistician involved in the previous paper from the
United Kingdom (on which current clinical policy is based) had taken the opposite view. ${ }^{2}$

As the table shows, analysis A included all children with meningococcal disease for whom data were available and estimated a small protective effect of penicillin. Analysis B, which included only children in whom the general practitioner had suspected a diagnosis of meningococcal disease, estimated a substantial (sixfold) reduction in survival.

\section{Simpson's paradox}

This rather frightening statistical effect-actually changing the direction of the estimated effect from benefit to harm-is related to Simpson's paradox (or the Yule-Simpson effect), first described in 1952. ${ }^{3}$ Simpson reported the seemingly impossible situation where the success of several subgroups seems to be reversed when the groups are combined. ${ }^{4}$
Editorial by Keeley, and pp 1295, 1299

Department of Primary Health Care, University of Oxford, Oxford OX3 7LF Rafael Perera senior research fellow in statistics

rafael.perera@ dphpc.ox.ac.uk 
Estimated increased odds of death in children with suspected meningococcal disease given penicillin before admission to hospital according to analysis chosen

\begin{tabular}{lccc} 
& Children included & Children excluded & Crude odds ratio \\
\hline Analysis A & 448 & 0 & 0.85 \\
\hline Analysis B & $152^{\star}$ & $290 \dagger$ & 5.96 \\
\hline
\end{tabular}

${ }^{\star}$ Excluded six children because we could not determine whether or not penicillin had been administered before admission.

tNone of excluded children received penicillin before admission so an odds ratio cannot be obtained for this group.

The reason for the paradox is the combination of two factors: an imbalance in the proportion of each subgroup receiving each intervention and a different event rate in each subgroup. This was the case with the penicillin data. To have a chance of being given penicillin, children had to be seen by a general practitioner who suspected meningococcal disease, and children who were seen by a general practitioner had a lower mortality $(18 \%)$ than those that were not (37\%).

Analysis A was based on all the children with meningococcal disease in our study. It replicated previous work and was therefore reassuring. But on reflection and discussion with the clinicians, I realised it transgressed one basic statistical principle-it included in the analysis children who had no chance of receiving penicillin before admission. I therefore excluded the children who had not been seen by a general practitioner or in whom he or she had not diagnosed meningococcal disease (analysis $\mathrm{B}$ in the table). This analysis produced the evidence of substantially increased mortality.

Defining the population highlighted two important sources of confounding: the fast progression of the disease and the lack of specific signs and symptoms early in the illness. ${ }^{5}$ The analysis reported is based on a population composed only of children with a more slowly progressive disease (who had time to see their doctor) and in whom the signs and symptoms were specific enough for a diagnosis. The 158 children in whom the general practitioner diagnosed meningococcal disease were at a later stage of their illness than the 166 who also saw their general practitioner but were not so diagnosed (median time from onset of illness to consultation $14 v 8$ hours). Furthermore, if the critical decision to administer penicillin in the 158 children is associated with severity of disease at the time (for example, more ill, higher chance that penicillin will be given) then the effect would be biased in the direction of penicillin causing harm. I thought it essential to adjust for severity of disease at the point at which the decision to give penicillin had been made.

Unfortunately, the limited data available made this difficult. The only validated measure of severity collected, GMSPS score, was assessed at admission to hospital-by which time penicillin is likely to have had an effect. Though severity scores at the time of diagnosis from the general practitioner's notes were obtained, recording was incomplete. Nevertheless I used this partial assessment of severity at diagnosis, together with other recorded variables that are believed to be associated with mortality (such as type of disease), to obtain an adjusted effect of penicillin on mortality. Having adjusted with these variables I would have expected the association between penicillin and mortality to get weaker or disappear. The estimate adjusted for severity, however, showed a further increase in the association between penicillin and mortality (adjusted odds ratio 7.45, 95\% confidence interval 1.47 to 37.67$)$. The question still in my mind is whether the variables used did truly adjust for severity of disease.

I decided to write this commentary to highlight the major impact that simple statistical decisions can have on the results of clinical research; to increase awareness of the possibility of Simpson's paradox, particularly in observational data of this nature; and to emphasise the importance of not assuming that strong associations are necessarily causal.

I thank Anthony Harnden, Richard Mayon-White, Matthew Thompson, and David Mant for help in preparing the manuscript.

Competing interests: None declared.

1 Harnden A, Ninis N, Thompson M, Perera R, Levin M, Mant D, et al. Parenteral penicillin for children with meningococcal disease before hospital admission: case-control study. BMJ 2006:332:1295-7.

2 Cartwright K, Reilly S, White D, Stuart J. Early treatment with parenteral penicillin in meningococcal disease. BMJ 1992;305:143-7.

3 Simpson EH. The interpretation of interaction in contingency tables. $J$ Roy Stat Soc 1951;13:238-41.

4 Julious SA, Mullee MA. Confounding and Simpson's paradox. BM/ 1994;309:1480-1.

5 Thompson MJ, Ninis N, Perera R, Mayon-White R, Phillips C, Bailey L, et al. Clinical recognition of meningococcal disease in children and adolescents. Lancet 2006;367:397-403.

(Accepted 18 April 2006)

\section{Corrections and clarifications}

Cannabis intoxication and fatal road crashes in France: population based case-control study

The authors of this paper, Bernard Laumon and colleagues (BMJ 2005;331:1371-4), have alerted us to some wrong values in two of the tables in the full version (see bmj.com). In table 1 the correct values for Friday and Saturday night respectively are: all drivers, $532(5.4 \%)$ and $649(6.6 \%)$; positive for cannabis, $67(9.8 \%)$ and $92(13.5 \%)$; and positive for alcohol, $259(12.4 \%)$ and 355 (16.9\%). In table 4 the correct values for Friday and Saturday night respectively are: cases, $410(6.1 \%)$ and $523(7.7 \%)$; controls, $122(4.1 \%)$ and $126(4.2 \%)$. The authors confirm that these changes do not affect the conclusions of the paper.

Editor's choice: Improving on improvement

In her Editor's choice of 6 May (BMJ 2006;332), Fiona Godlee mentioned Frank Davidoff's draft publication guidelines for quality improvement reports. However, she mistakenly named his coauthor as David Batalden, whereas his real name is Paul Batalden.

Active and passive smoking and development of glucose intolerance among young adults in a prospective cohort: CARDIA study

In the methods section of this paper by Houston and colleagues (BMJ 2006;332:1064, 6 May), the definition of never smokers with passive smoke exposure should include all never smokers who reported having had passive smoke exposure or (not "and" as mistakenly stated) who had detectable serum cotinine concentrations $(1-15 \mathrm{ng} / \mathrm{ml})$. 